\title{
Resultados funcionais e radiológicos do tratamento cirúrgico da luxação acromioclavicular aguda com âncoras e fixação clavículo-escapular*
}

\section{Functional and Radiological Outcomes of the Surgical Treatment of Acute Acromioclavicular Dislocation with Anchors Associated with Clavicle and Scapula Fixation}

\author{
Adriano Fernando Mendes Júnior ${ }^{10}$ José da Mota Neto ${ }^{1}$ Darlan Malba Dias ${ }^{2}$ \\ Leandro Furtado de Simoni ${ }^{3}$ Elmano de Araújo Loures ${ }^{1}$ Pedro José Labronici ${ }^{4}$
}

\footnotetext{
${ }^{1}$ Departamento de Ortopedia e Traumatologia, Hospital Universitário, Universidade Federal de Juiz de Fora, Juiz de Fora, MG, Brasil

2 Serviço de Ortopedia e Traumatologia, Hospital Santa Luzia, Brasília, DF, Brasil

${ }^{3}$ Hospital Maternidade Therezinha de Jesus, Juiz de Fora, MG, Brasil

${ }^{4}$ Departamento de Cirurgia, Universidade Federal Fluminense (UFF),

Niterói, RJ, Brasil
}

Endereço para correspondência Adriano Fernando Mendes Júnior,

Rev Bras Ortop 2019;54:649-656.

MD, MSc, Rua Sampaio 468, apto. 1.402, Juiz de Fora, MG, 36010-360,
Brasil (e-mail: adrianofmjr@yahoo.com.br).

\begin{abstract}
Resumo
Palavras-chave

- articulação acromioclavicular

- resultado do tratamento

- âncoras de sutura

Objetivo Avaliar os resultados clínicos, radiológicos, e funcionais do tratamento cirúrgico da luxação acromioclavicular aguda, utilizando a técnica de sindesmopexia coracoclavicular com duas âncoras metálicas, fixação temporária clavículo-escapular, e transferência do ligamento coracoacromial.

Métodos Estudo observacional longitudinal com trinta pacientes com diagnóstico de luxação acromioclavicular aguda submetidos à cirurgia, com seguimento mínimo de seis meses, avaliados clínica, radiograficamente, e pelos escores de University of California at Los Angeles (UCLA), Disabilities of the Arm, Shoulder and Hand (DASH) e Constant-Murley.

Resultados Os valores médios dos escores foram: $U C L A=32$; $D A S H=11,21$; e Constant-Murley $=86,93$, com resultados satisfatórios acima de $80 \%$. Os resultados insatisfatórios foram relacionados à dor acromioclavicular, a testes de impacto subacromial positivos, e aos pacientes de faixa etária mais elevada $(p<0,05)$. Radiologicamente, valores maiores em razão da distância coracoclavicular do ombro operado, comparado com o ombro normal, foram relacionados a piores resultados, embora sem significância estatística. Não foi observada associação entre os resultados
\end{abstract}

Trabalho realizado no Hospital Universitário, Universidade Federal de Juiz de Fora, Juiz de Fora, MG, Brasil

recebido

15 de Abril de 2018

aceito

04 de Junho de 2018
DOI https://doi.org/

10.1055/s-0039-1697020. ISSN 0102-3616.
Copyright $\odot 2019$ by Sociedade Brasileira License terms de Ortopedia e Traumatologia. Published by Thieme Revinter Publicações Ltda, Rio de Janeiro, Brazil 


\begin{abstract}
Keywords

- acromioclavicular joint

- treatment outcome

- suture anchors

Objective To evaluate the clinical, radiological and functional results of the surgical treatment of acute acromioclavicular dislocation using a coracoclavicular fixation technique with two metal anchors, temporary clavicle and scapula fixation, and transfer of the coracoacromial ligament.

Methods Longitudinal observational study of 30 patients with diagnoses of acute acromioclavicular dislocation, who were submitted to surgical treatment with a minimum follow-up of six months, and who were evaluated clinically, radiologically, and by the University of California at Los Angeles (UCLA), the Disabilities of the Arm, Shoulder and Hand (DASH), and the Constant-Murley functional scores.

Results The mean values of the scores were: $\mathrm{UCLA}=32$; $\mathrm{DASH}=11.21$; and ConstantMurley $=86.93$, with satisfactory results higher than $80 \%$ The unsatisfactory results were associated with acromioclavicular pain on palpation , positive subacromial impingement tests, and older age group, presenting statistical significance $(p<0,05)$. Radiologically, igher values on account of the coracoclavicular distance from the operated shoulder compared to the normal shoulder were related to worse outcomes, but with no statistically significant difference. No associations were found between the results of the functional outcomes and the variables degree of the injury, coracoacromial ligament transfer, clinical impression of loss of reduction, and scapulothoracic dyskinesia.

Conclusion The technique used provides an efficient fixation, with a high level of satisfaction according to the UCLA, Constant-Murley and DASH scores; moreover, it has a low complication rate, despite the high rate of residual radiological subluxation.
\end{abstract}

dos escores funcionais e as variáveis grau da lesão, transferência do ligamento coracoacromial, impressão clínica de perda de redução, e discinesia escapulo-torácica. Conclusão A técnica utilizada propicia uma fixação eficiente, com alto índice de satisfação segundo os escores de UCLA, Constant-Murley e DASH. Observou-se baixo índice de complicações apesar da frequência elevada de subluxação acromioclavicular radiológica residual.

\section{Introdução}

A luxação acromioclavicular (LAC) é uma lesão frequente, que ocorre geralmente após queda ao solo, com trauma direto sobre o ombro, e o braço em adução. ${ }^{1}$ Representa entre $9 \%$ e $12 \%$ das lesões na cintura escapular, sendo mais frequente em adultos jovens, atletas, e cinco vezes mais comum em homens do que em mulheres. ${ }^{1,2}$ A maioria das lesões do complexo acromioclavicular (AC) são incompletas, comprometendo principalmente os ligamentos ACs. ${ }^{1} \mathrm{Em}$ casos de trauma de maior intensidade deste complexo, ocorrem também lesões nos ligamentos coracoclaviculares (CCs) e na fáscia deltotrapezoidal, ambos estabilizadores da articulação $\mathrm{AC}(\mathrm{AAC})^{2}$

As lesões ACs foram originalmente descritas por Tossy et $\mathrm{al}^{2}$ em graus I, II e III, e foram posteriormente modificadas por Rockwood e Williams, ${ }^{3}$ que descreveram os graus IV, V e VI. Esta classificação apresenta boa reprodutibilidade entre ortopedistas, ${ }^{3}$ e também é utilizada para guiar a indicação de tratamento clínico ou cirúrgico. Segundo Rockwood e Williams, ${ }^{3}$ o tratamento cirúrgico está preconizado para os graus IV, V e VI, e em alguns casos do grau III. Os casos de graus I e II têm tratamento conservador. Há controvérsia sobre o tratamento ideal da LAC, principalmente em relação ao grau III. $^{4}$ Entre as opções de tratamento cirúrgico, são descritas a fixação da AAC com fios ou placa, associada ou não à transferência do ligamento coracoacromial (CA), fixação CA com parafuso CC ou âncoras CCs, fixação temporária com fios de Kirschner, e reconstruções ligamentares. ${ }^{3-5}$

Não há consenso quanto ao tratamento desta lesão. ${ }^{5}$ As modificações das técnicas que aproximam a clavícula do processo coracoide (sindesmopexia) passaram do uso de parafuso ou amarrilho subcoracoide para o uso de âncoras e materiais como o Endobutton (Smith \& Nephew, Londres, Reino Unido), que podem ser realizados por via aberta, minimamente invasiva ou artroscópica. A opção para o tratamento cirúrgico da LAC aguda, com estabilização CC utilizando duas âncoras, apresenta a vantagem de mimetizar a origem anatômica dos ligamentos CCs e suas inserções na clavícula; ${ }^{6}$ outra vantagem é a utilização de um implante acessivel para os ortopedistas brasileiros.

O objetivo deste estudo é avaliar os resultados clínicos, radiológicos e funcionais do tratamento cirúrgico da LAC aguda, utilizando a técnica de sindesmopexia CC com duas âncoras metálicas e fixação temporária clavículo-escapular. 


\section{Materiais e Métodos}

Este estudo foi aprovado pelo Comitê de Ética em Pesquisa da instituição (CAAE 58252216.0.0000.5133), e todos os indivíduos selecionados concordaram em participar por meio da assinatura do termo de consentimento livre e esclarecido (TCLE). Trata-se de um estudo longitudinal observacional de pacientes com LAC aguda, tratados cirurgicamente por três dos cirurgiões participantes do estudo, pela técnica de sindesmopexia CC com duas âncoras metálicas e fixação temporária clavículo-escapular, em hospitais terciários, no período de janeiro de 2011 a março de 2016. A amostra estudada compreendeu 41 indivíduos operados por conta de LAC aguda de graus III, IV e V de Rockwood e Williams. ${ }^{3}$ Os critérios de inclusão foram: idade maior do que 18 anos, cirurgia realizada 6 meses ou mais antes da data de avaliação, e tratamento cirúrgico de LAC aguda pela técnica citada considerando, para efeito temporal, o período de até 3 semanas como LAC aguda. ${ }^{7}$ Os critérios de exclusão foram: histórico de LAC pregressa no mesmo membro, cirurgias prévias no mesmo ombro, e incapacidade de responder aos questionários de avaliação funcional ou de comparecer à reavaliação ambulatorial. Dos 41 pacientes, 1 estava em coma devido a complicações não relacionadas ao procedimento cirúrgico; 4 se recusaram a participar do estudo; 2 não se enquadravam no critério de tratamento de LAC aguda; e 4 não foram localizados para avaliação. A amostra final foi de 30 participantes, cujos prontuários foram analisados, e os pacientes foram submetidos a avaliação funcional e radiológica.

O desfecho primário do estudo foi o escore funcional da University of California at Los Angeles (UCLA), ${ }^{8}$ seguido dos escores Disabilities of the Arm, Shoulder and Hand (DASH $)^{9} \mathrm{e}$ de Constant-Murley, ${ }^{10}$ e os resultados encontrados foram classificados como satisfatórios ou insatisfatórios. ${ }^{8,11}$ Os desfechos secundários avaliados foram: a transferência do ligamento CA no procedimento; complicações decorrentes do ato cirúrgico; média de idade dos pacientes; dados do exame clínico (impressão clínica da perda de redução da LAC, discinesia escápulo-torácica [DET], dor à palpação AC, manobras de impacto subacromial e AC, como os testes de Neer, Hawkings-Kenedy, Yokun e cross-body aduction); ${ }^{12}$ e avaliação radiológica (perda de redução, degeneração AC).

O diagnóstico de LAC, bem como a classificação segundo os critérios de Rockwood e Williams, ${ }^{3}$ foi feito radiologicamente nas incidências anteroposterior (AP) e Zanca para clavícula e perfil axilar do ombro. ${ }^{13} \mathrm{~A}$ avaliação radiológica também utilizou um índice como medida comparativa da distância coracoclavicular (dCC) dos lados operado e normal (dCC_O/N), na radiografia da clavícula nas incidências AP e Zanca, dividindo-se o valor da dCC do lado operado (dCC_O) pela do lado normal (dCC_N), como demonstrado na - Figura 1. Foram adotados ainda os critérios radiológicos conforme Rush et $\mathrm{al}^{14}$ para avaliação da redução da LAC pós-cirurgia, considerando-se perda da redução pós-cirúrgica o aumento da dCC_O/N superior ou igual a 2, e a subluxação residual como dCC_O/N entre 1 e 2.

0 procedimento foi realizado com o paciente na posição de cadeira de praia, sob bloqueio do plexo braquial e anestesia geral. Realizou-se incisão tipo sabre no sentido AP, iniciando a $1 \mathrm{~cm}$ medial à $A A C$, da borda posterior da clavícula até $1 \mathrm{~cm}$ superior à borda superior do processo coracoide. Quando intacta, a fáscia deltotrapezoidal foi incisada paralela às suas fibras. Em alguns pacientes, a critério do cirurgião, foi identificado o ligamento $C A$, que foi dissecado até a sua origem acromial e, então, seccionado e reparado para transferência ao terço lateral da clavícula, em fixação transóssea. Por meio de dissecação, a base do processo coracoide foi exposta, permitindo a inserção de duas âncoras metálicas de $4 \mathrm{~mm}$ cada, com 1 ou 2 fios de suturas não absorvíveis, adaptada conforme a técnica de Dal Molin et al. ${ }^{6}$ As suturas foram passadas através de 2 furos feitos na clavícula com broca de $2,0 \mathrm{~mm}$, com o objetivo de reproduzir a inserção anatômica dos ligamentos CCs: um orifício posteromedial para o ligamento conoide e, a $1 \mathrm{~cm}$ lateral a este, um orifício anterolateral de mesmo tamanho, para o trapezoide, a uma distância de $1 \mathrm{~cm}$ da AAC. Antes da aproximação CC com as suturas de ancoragem, a AAC foi reduzida anatomicamente: enquanto o assistente executava a manobra de estabilização da escápula em retração e abaixamento da

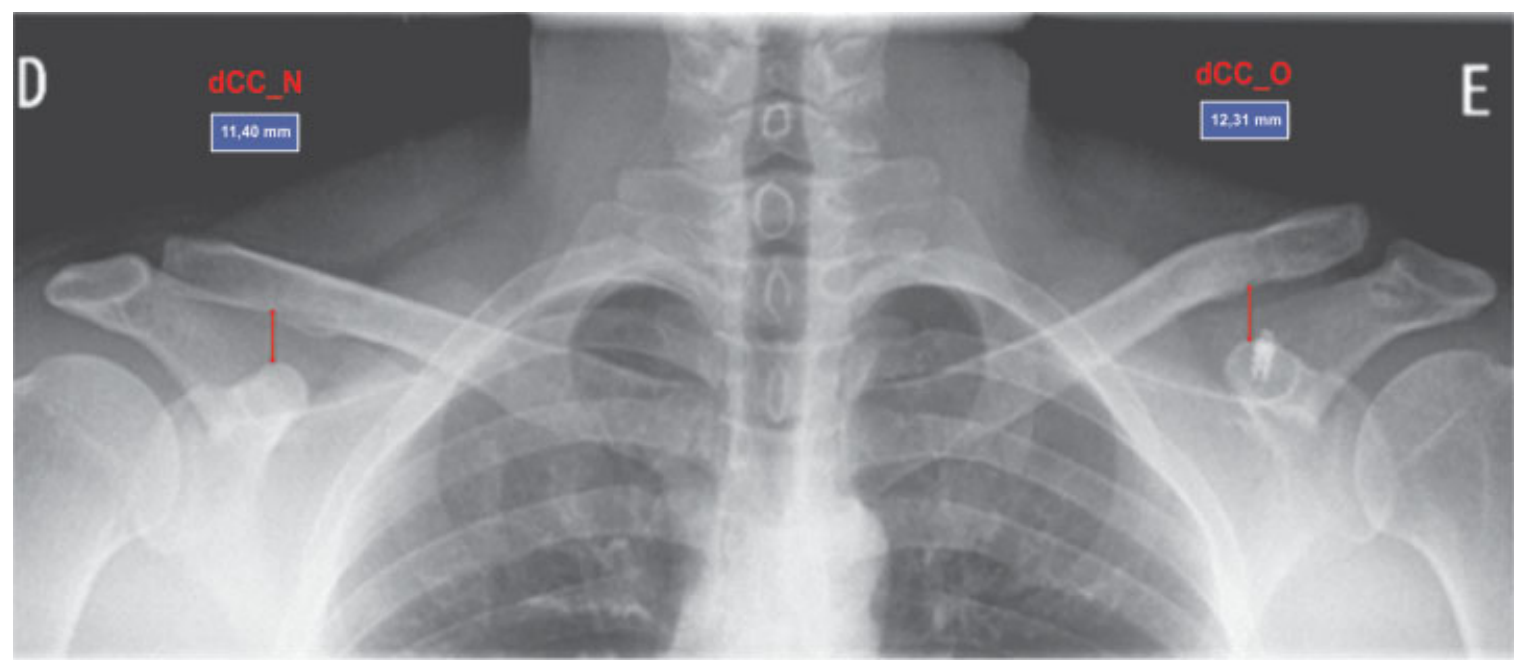

Fig. 1 Cálculo da distância coracoclavicular dos lados operado e normal (dCC_O/N: 12,31/11,40=1,07). Paciente com subluxação residual de $7 \%$. 

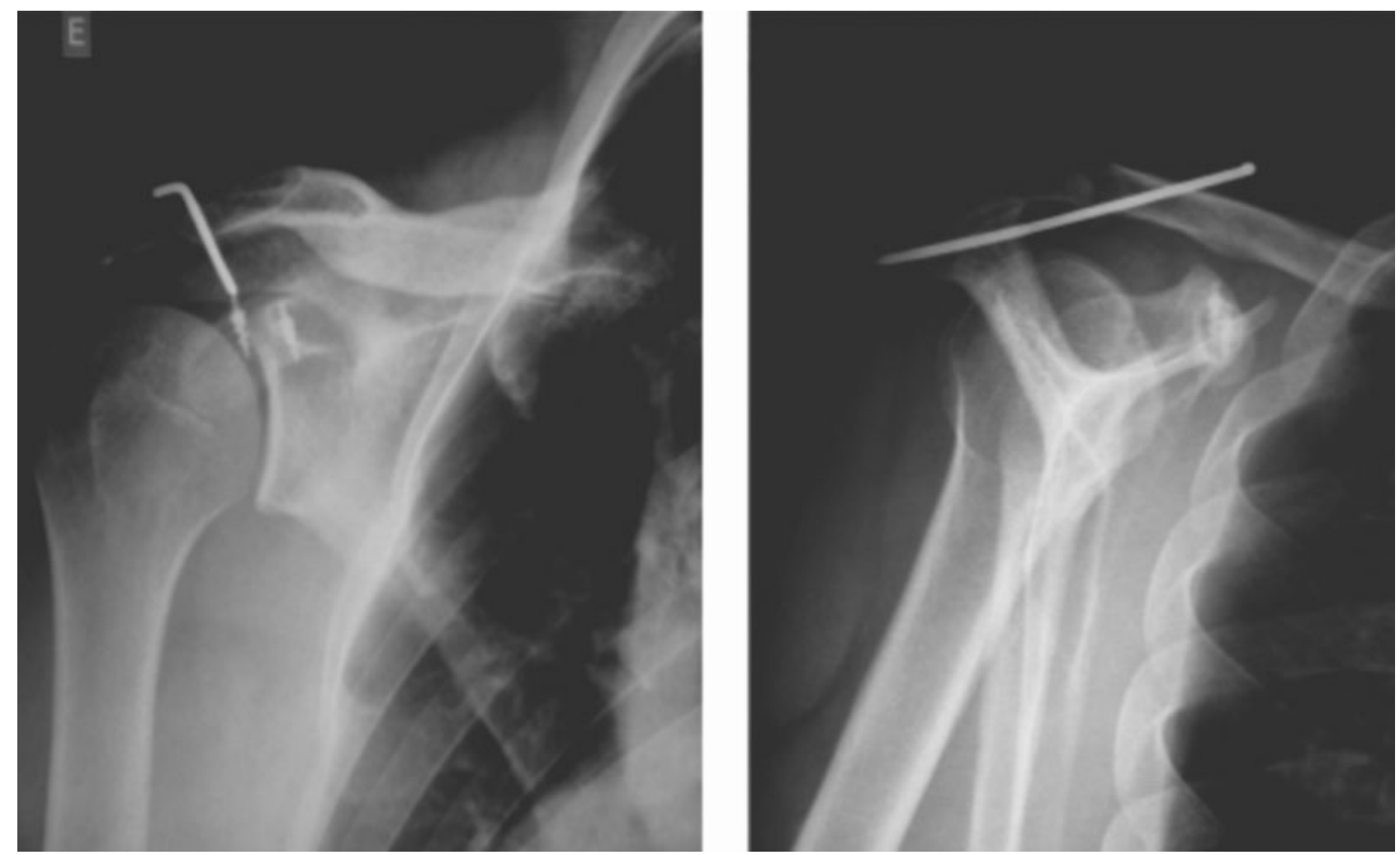

Fig. 2 Radiografia em incidência anteroposterior do ombro e perfil da escápula no pós-operatório, evidenciando as âncoras no processo coracoide e o fio de Kirschner de fixação provisória entre a clavícula e a espinha da escápula.

clavícula, o cirurgião realizava a fixação clavículo-espinal dirigindo o fio de Kirschner a 2,5 mm da borda anterossuperior do terço lateral da clavícula à base da espinha escapular, segundo a técnica descrita por Carrera et $\mathrm{al}^{15}$ (- Figura 2). As suturas das âncoras, previamente passadas através dos orifícios na clavícula, foram amarradas, e o ligamento CA, quando transferido, foi tensionado. A fáscia deltotrapezial foi cuidadosamente suturada, e o fio de Kirschner, sepultado sob a fáscia, realizando-se, assim, o fechamento final por planos.

No pós-operatório, o membro foi imobilizado em tipoia de abdução durante 6 semanas. 0 paciente foi orientado a começar imediatamente exercícios de flexo/extensão do cotovelo, e de suave rotação medial e lateral do ombro. A elevação do ombro acima de 45 graus foi desencorajada para evitar quebra ou migração do fio de Kirschner clavículoescapular, ou afrouxamento da sua inserção. Após seis semanas, o fio de Kirschner foi removido em centro cirúrgico, sob anestesia local e sedação, e o paciente foi autorizado a realizar todos os movimentos do ombro e a começar a reabilitação fisioterápica. $\mathrm{O}$ retorno à prática de esportes de contato foi permitido após três meses de cirurgia.

A análise estatística, com as variáveis quantitativas, foi descrita pela média e pelo desvio padrão, e as variáveis qualitativas, por frequência absoluta e porcentagens. Para testar diferenças entre os grupos com relação às variáveis quantitativas, foi utilizado o teste U de Mann-Whitney. O tamanho do efeito foi avaliado pelo $d$ de Cohen ${ }^{16}$, utilizando-se o desvio padrão ponderado e adotando-se a seguinte classificação para interpretação: pequeno $=0,20$ 0,49 ; moderado $=0,50-0,79$; elevado $\geq 0,80 .{ }^{16}$ Para testar diferenças entre proporções, utilizou-se o teste Exato de Fisher. Neste caso, o tamanho do efeito foi avaliado pelo $V$ de Cramér, adotando-se a seguinte classificação para interpretação: pequeno $=0,10-0,29$; moderado $=0,30-0,49$; elevado $\geq 0,50 .{ }^{16}$ Todas as análises foram feitas no software estatístico International Businness Machines Statistical Package for the Social Sciences (IBM SPSS, IBM Corp., Armonk, NY, EUA), versão 24.0. O nível de significância estatística foi estabelecido como $p<0,05$. Para o cálculo do tamanho amostral, quando necessário, foi utilizado o software $\mathrm{G}^{*}$ Power 3.1(Universität Düsseldorf, Düsseldorf, Renânia do Norte-Vestfália, Alemanha). ${ }^{17}$

\section{Resultados}

Um total de 30 indivíduos preencheram os critérios de inclusão, sendo a maioria do gênero masculino $(n=28$; 93,3\%). O seguimento médio foi de 18 meses ( 7 a 40 meses), a média de idade dos pacientes foi de 40 anos $( \pm 12,7 ; 20$ a 71 anos), e 28 deles eram destros (93,3\%). 0 membro dominante foi acometido em 15 pacientes (50\%), e o tempo médio decorrido até a cirurgia foi de 6 dias $( \pm 6,4)$. As causas mais comuns foram queda ao solo $(n=9)$, queda de bicicleta $(\mathrm{n}=9)$ e queda de motocicleta $(\mathrm{n}=8)$. De acordo com a classificação de Rockwood e Williams, ${ }^{3}$ os pacientes foram classificados como grau III $(n=16)$, grau IV $(n=3)$ e grau V $(\mathrm{n}=11)$. A transferência do ligamento $\mathrm{CA}$ foi realizada em 17 pacientes (56,7\%). Um total de 5 casos (16,6\%) evoluíram com complicações pós-operatórias agudas, sendo: 1 caso de fio de Kirschner proeminente, que causou desconforto na região do 
ombro posterior; 1 caso de exteriorização do fio de Kirschner na sexta semana de pós-operatório, ambos solucionados com a remoção do fio na sexta semana após a cirurgia; 2 casos de infecção da ferida operatória tratados com antibióticos por via oral por 10 dias; e 1 caso de infecção profunda, que necessitou lavagem e desbridamento cirúrgico, seguido de antibióticos endovenosos por 2 semanas.

Na avaliação clínica, 6 pacientes $(20,0 \%)$ apresentaram dor à palpação AC, e 10 pacientes $(30,0 \%)$ apresentaram DET. Testes de impacto subacromial positivos foram observados em 6 pacientes (20\%), e a impressão clínica da perda da redução após a cirurgia, avaliada pelo observador, foi percebida em 9 pacientes (30\%). O índice de satisfação espontânea com o tratamento foi de $96,87 \%$.

$\mathrm{Na}$ avaliação funcional, foram obtidos os valores médios finais (desvio padrão) dos escores: $\mathrm{UCLA}=32( \pm 6,33)$; DASH $=11,21( \pm 20,18)$; e Constant-Murley $=86,93( \pm 20,34)$. Na classificação dos resultados dos escores como satisfatórios ou não, encontrou-se que $86,7 \%$ da amostra tiveram UCLA satisfatório ( $>27$ ); $83,3 \%$ tiveram DASH satisfatório ( $<20$ ); e 80,0\% tiveram Constant-Murley satisfatório ( $>79$ ).

Na avaliação radiológica, a degeneração AC foi observada em 4 pacientes (13,3\%). Segundo os critérios de Rush et al, ${ }^{14} 2$ casos (6,6\%) apresentaram perda da redução na incidência AP, e 4 casos (13,3\%), na incidência Zanca. Subluxações residuais foram observadas em 24 pacientes (80\%) na incidência AP, e em 20 pacientes (67\%) na Zanca. Apenas 4 pacientes (13\%) na incidência AP e 6 pacientes (20\%) na Zanca mantiveram a redução do lado oposto.

A comparação entre as características clínicas e os escores UCLA, DASH e Constant-Murley está apresentada na - Tabela 1. Observou-se que os pacientes com resultados funcionais insatisfatórios apresentaram dor na palpação AC, testes de impacto subacromial positivos, e maior média de idade quando comparados aos pacientes com resultados funcionais satisfatórios. Pacientes com mais de 50 anos apresentaram piores resultados funcionais. Para essas variáveis, as relações e diferenças observadas foram de elevada magnitude $(d>0,80 ; V>0,50)$. O grau da LAC e a DET não estão associados aos resultados funcionais avaliados pelos escores de UCLA, DASH e Constant-Murley ( $p>0,05)$.

Radiologicamente, observou-se que a degeneração AC foi mais frequente nos pacientes com resultados insatisfatórios avaliados pelo UCLA, embora este resultado tenha ficado no limiar de significância $(p=0,07)$. Considerando a prevalência de $15 \%$ de resultados insatisfatórios no UCLA, estima-se um tamanho de amostra de 45 pacientes, para que seja encontrada associação estatisticamente significativa entre a degeneração acromioclavicular observada na radiografia e o resultado funcional avaliado pelo UCLA. ${ }^{17}$

A medida da razão da dCC_O/N nas incidências radiológicas em AP e Zanca foi correlacionada com os dados clínicos e os escores funcionais. Verificou-se que os pacientes com resultados insatisfatórios nos escores funcionais apresentaram maiores valores na razão da dCC_O/N, embora isso não tenha sido estatisticamente significativo $(p>0,05)$; porém, o tamanho do efeito observado no DASH e no Constant-Murley para a associação da razão da dCC_O/N na incidência Zanca foi de moderada magnitude $(d=0,66$ e $d=0,56$, respectivamente). Como cálculo amostral, considerando a proporção de $17 \%$ de pacientes com resultados funcionais insatisfatórios, seriam necessários 204 pacientes (30 pacientes com resultados insatisfatórios) para se observar significância estatística entre o aumento da razão da dCC_O/N e piores resultados nos questionários funcionais. ${ }^{17}$

Não foi observada diferença estatisticamente significativa na razão da dCC_O/N nas incidências AP e Zanca, em relação ao grau da lesão $(p>0,05)$, à transferência do ligamento CA

Tabela 1 Associação entre a avaliação clínica e os escores funcionais UCLA, DASH e Constant-Murley

\begin{tabular}{|c|c|c|c|c|c|c|c|c|c|}
\hline \multirow[b]{2}{*}{$\begin{array}{l}\text { Variáveis/ } \\
\text { Categorias }\end{array}$} & \multicolumn{3}{|l|}{ UCLA } & \multicolumn{3}{|l|}{ DASH } & \multicolumn{3}{|c|}{ Constant-Murley } \\
\hline & $\begin{array}{l}S \\
(n=26)\end{array}$ & $\begin{array}{l}\text { I } \\
(n=4)\end{array}$ & $\begin{array}{l}\text { Valor } \\
\text { de } p\end{array}$ & $\begin{array}{l}S \\
(n=25)\end{array}$ & $I(n=5)$ & $\begin{array}{l}\text { Valor } \\
\text { de } p\end{array}$ & $\begin{array}{l}S \\
(n=24)\end{array}$ & $\begin{array}{l}\text { I } \\
(n=6)\end{array}$ & Valor de $p$ \\
\hline \multicolumn{10}{|l|}{ Grau da LAC } \\
\hline III $(n=16)$ & $87,50 \%$ & $12,50 \%$ & 1 & $81,30 \%$ & $18,80 \%$ & 1 & $81,20 \%$ & $18,80 \%$ & 1 \\
\hline IV e $V(n=14)$ & $85,70 \%$ & $14,30 \%$ & & $85,70 \%$ & $14,30 \%$ & & $78,60 \%$ & $21,40 \%$ & \\
\hline \multicolumn{10}{|l|}{ Dor AC } \\
\hline Não $(n=24)$ & $100,00 \%$ & $0,00 \%$ & $0,001^{*}$ & $95,80 \%$ & $4,20 \%$ & $0,003^{*}$ & $91,70 \%$ & $8,30 \%$ & $0,007^{*}$ \\
\hline $\operatorname{Sim}(n=6)$ & $33,30 \%$ & $66,70 \%$ & & $33,30 \%$ & $66,70 \%$ & & $33,30 \%$ & $66,70 \%$ & \\
\hline \multicolumn{10}{|l|}{ Testes de impacto } \\
\hline Não $(n=24)$ & $100,00 \%$ & $0,00 \%$ & $0,001^{*}$ & $100,00 \%$ & $0,00 \%$ & $<0,001^{*}$ & $95,80 \%$ & $4,20 \%$ & $<0,001^{*}$ \\
\hline $\operatorname{Sim}(n=6)$ & $33,30 \%$ & $66,70 \%$ & & $16,70 \%$ & $83,30 \%$ & & $16,70 \%$ & $83,30 \%$ & \\
\hline $\begin{array}{l}\text { Idade } \\
\text { (média } \pm \text { desvio } \\
\text { padrão em anos) }\end{array}$ & $38,2 \pm 11,7$ & $56,7 \pm 3,2$ & $0,005^{*}$ & $\begin{array}{l}38,0 \pm \\
11,9\end{array}$ & $\begin{array}{l}54,0 \pm \\
6,7\end{array}$ & $0,006^{*}$ & $\begin{array}{l}37,4 \pm \\
11,7\end{array}$ & $\begin{array}{l}53,8 \pm \\
6,0\end{array}$ & $0,002^{*}$ \\
\hline
\end{tabular}

Abreviaturas: AC, acromioclavicular; DASH, Disabilities of the Arm, Shoulder and Hand; I, insatisfatório; LAC, luxação acromioclavicular; S, satisfatório; UCLA, University of California at Los Angeles.

Notas: Porcentagens: em relação às linhas; valor de $p$ : teste Exato de Fisher e teste $\mathrm{U}$ de Mann-Whitney; ${ }^{*}$ diferença significativa: $p<0,05$. 
Tabela 2 Relação entre valores médios dos escores funcionais e razões da dCCO_N nas radiografias AP e Zanca

\begin{tabular}{|l|l|l|l|l|l|l|l|}
\hline Variável & $\mathbf{n}$ & DASH & Valor de $\boldsymbol{p}$ & Constant-Murley & Valor de $\boldsymbol{p}$ & UCLA & Valor de $\boldsymbol{p}$ \\
\hline Razão da dCC_O/N AP & & & $0,92^{*}$ & & 0,41 & & $0,84^{*}$ \\
\hline$<1,5$ & 23 & $11,9 \pm 21,1^{\mathrm{a}}$ & & $87,8 \pm 19,7^{\mathrm{a}}$ & & $32,0 \pm 6,1^{\mathrm{a}}$ & \\
\hline$\geq 1,5$ & 7 & $8,9 \pm 18,0^{\mathrm{a}}$ & & $84,0 \pm 23,7^{\mathrm{a}}$ & & $31,8 \pm 7,4^{\mathrm{a}}$ & \\
\hline Razão dCC_O/N Zanca & & & $0,22^{*}$ & & 0,19 & & $0,61^{*}$ \\
\hline$<1,5$ & 18 & $9,3 \pm 19,9^{\mathrm{a}}$ & & $89,7 \pm 18,5^{\mathrm{a}}$ & & $32,7 \pm 4,8^{\mathrm{a}}$ & \\
\hline$\geq 1,5$ & 12 & $14,1 \pm 21,1^{\mathrm{a}}$ & & $82,8 \pm 23,0^{\mathrm{a}}$ & & $30,9 \pm 8,2^{\mathrm{a}}$ & \\
\hline
\end{tabular}

Abreviaturas: AP, anteroposterior; DASH, Disabilities of the Arm, Shoulder and Hand; dCC_O/Nn distância coracoclavicular dos lados operado e normal; UCLA, University of California at Los Angeles.

Notas: ${ }^{a}$ Média \pm desvio padrão; *valor de $p$ calculado pelo teste de Mann-Whitney.

$(p>0,05)$, e à impressão clínica de perda da redução $(p>0,05)$. Para avaliar a relação entre o valor de subluxação radiológica e os aspectos clínicos e funcionais, adotou-se o ponto de corte de 1,5 na razão da dCC_O/N. Não houve associação estatisticamente significativa com os escores funcionais (-Tabela 2; $p>0,05$ ).

Por fim, a transferência do ligamento CA no ato cirúrgico não apresentou diferença nos escores funcionais, e não se mostrou relacionada à impressão clínica de perda da redução e à razão da dCC_O/N nas incidências AP e Zanca.

\section{Discussão}

O objetivo do tratamento da LAC aguda é obter uma redução que permita a cicatrização das partes moles e a recuperação da função prévia da articulação. No entanto, os inúmeros procedimentos descritos na literatura comprovam a falta de consenso em relação ao método ideal. ${ }^{18}$ No presente estudo, os valores médios dos escores foram: UCLA $=32( \pm 6,33)$; $\mathrm{DASH}=11,21 \quad( \pm 20,18) ; \quad$ e Constant-Murley $=86,93 \quad( \pm-$ 20,34), com satisfação geral de $96,87 \%$ (todos menos 1 paciente da amostra). Algarín et al ${ }^{19}$ avaliaram 42 pacientes tratados com uma técnica minimamente invasiva, e obtiveram $86 \%$ de resultados satisfatórios, segundo o escore UCLA.

Existem diversas opções cirúrgicas para o tratamento da LAC, e elas estão didaticamente divididas entre técnicas de fixação CCs flexível (como os amarrilhos CCs ou a fixação com âncoras) ou rígida (como os parafusos CCs ou as placas tipo gancho). As opções ditas flexíveis são consideradas mais biológicas, pois proporcionam o movimento da clavícula em relação ao acrômio, e não necessitam da retirada dos implantes. A fixação CC com parafuso gera rigidez excessiva na AAC, podendo levar à quebra do implante, osteólise acromial ou clavicular, perda da redução, além da necessidade de novo procedimento para retirada. ${ }^{5} \mathrm{~A}$ utilização da placa gancho tem taxa elevada de complicações, incluindo osteólise acromial ou fratura, bursite subacromial, e osteoartrite. ${ }^{20,21}$ Segundo Koukakis et $\mathrm{al}^{20}$, a retirada do implante transfere a responsabilidade pela manutenção da redução unicamente aos tecidos cicatrizados, favorecendo a recorrência.

Mesmo as opções ditas flexíveis não são isentas de riscos. De acordo com Baker et al ${ }^{22}$, a cerclagem subcoracoide com fios de alta resistência pode causar erosão óssea local e manter a subluxação anterior da clavícula, o que tem demonstrado ser causa de dor, artrite e diminuição da força. A fixação da clavícula ao processo coracoide com âncoras de sutura e o uso de pino metálico temporário, entre a clavícula e a espinha da escápula, apresenta uma alternativa para evitar as complicações e simplificar a fixação temporária. ${ }^{15}$ Nos pacientes deste estudo, foram utilizadas âncoras CCs como procedimento principal, e a fixação clavículo-escapular temporária como método auxiliar. Segundo Tamaoki et $\mathrm{al}^{23}$, em estudo transversal com ortopedistas brasileiros sobre o tratamento de LAC, nos casos cirúrgicos de grau III, $63 \%$ dos entrevistados utiliza a fixação CC em seus pacientes, enquanto $51 \%$ prefere a técnica de fixação transarticular AC.

A fixação da clavícula na espinha da escápula foi realizada poupando a AAC de uma nova agressão, e visando evitar complicações, como a dor residual na AAC. Eskola et al, ${ }^{24} \mathrm{em}$ estudo randomizado de tratamento cirúrgico de LAC que comparou a fixação CC com parafuso e fios metálicos transarticulares AC, mostraram melhores resultados com a utilização de fios metálicos transarticulares. No entanto, a técnica está associada a uma taxa elevada de complicações, incluindo infecção, perda de redução, desenvolvimento de osteoartrite AC, além de quebra e migração do fio metálico. Esse estudo mostra benefícios na fixação temporária entre a clavícula e a espinha da escápula, pois evita o afrouxamento das suturas de ancoragem nas primeiras semanas após a cirurgia, permitindo uma melhor cicatrização de tecidos moles e auxiliando na manutenção da redução da luxação após a remoção do fio de Kirschner.

O melhor método de tratamento para lesões de grau III é incerto. ${ }^{25}$ Alguns autores descrevem resultados similares de tratamento cirúrgico e não cirúrgico em casos de grau III, ${ }^{18}$ enquanto outros encontraram resultados insatisfatórios com o tratamento não cirúrgico, representado por dor residual e diminuição da força muscular da cintura escapular em até $50 \%$ dos casos, favorecendo a conduta cirúrgica como escolha nestes casos. ${ }^{26}$ Segundo Rasmont et $\mathrm{al}^{27}$, o tratamento conservador é a primeira escolha nestas lesões. Na amostra estudada, o percentual de satisfação dos pacientes com LAC de grau III encontrado foi de $100 \%$, e, na avaliação funcional, os resultados satisfatórios foram de $87,5 \%$ no UCLA, de $81,3 \%$ no DASH, e de $81,2 \%$ no Constant-Murley. Tais resultados demonstram que este método foi uma boa opção para esse 
grupo de pacientes, com altas taxas de satisfação ao final do acompanhamento.

Apesar de a literatura mostrar bons resultados funcionais na utilização das âncoras metálicas no coracoide como método de tratamento da LAC aguda, ${ }^{15,18,19}$ foram encontradas distinções nos resultados de acordo com a idade dos pacientes. Na amostra do presente estudo, houve diferença estatisticamente significativa, com piores resultados funcionais no UCLA ( $p=0,005)$, DASH $(p=0,006)$ e Constant-Murley $(p=0,002)$, em pacientes com idade média de $56,7( \pm 3,2)$, $54,0( \pm 6,7)$, e $53,8( \pm 6,0)$, respectivamente, sugerindo que o tratamento da LAC aguda por este método, nos pacientes dessa faixa etária, deve ser revisto. Estudos adicionais são necessários para elucidar a relação entre resultados funcionais insatisfatórios nos pacientes mais velhos.

Um dos procedimentos auxiliares, a transferência do ligamento CA, atua, em tese, como um reforço biológico para estabilização AC. Segundo Johansen et $\mathrm{al}^{5}$, o uso do ligamento CA tem limitações biomecânicas se for empregado de forma isolada no tratamento da instabilidade AC, necessitando ser utilizado simultaneamente com as reconstruções dos ligamentos CCs. Entretanto, não foram encontradas diferenças estatisticamente significativas em relação aos resultados funcionais entre os grupos que realizaram ou não a transferência adjuvante do ligamento CA.

A descrição, pelo observador, de perda de redução após a cirurgia ou de subluxações residuais ao exame radiológico é frequente. Segundo a literatura, a evidência de deformidade recorrente varia de $8 \%$ a $18 \% .{ }^{28-30} \mathrm{Na}$ técnica de reconstrução com âncoras, segundo Breslow et $\mathrm{al}^{31}$, um dos motivos para a perda da redução é o local impreciso da inserção das âncoras no coracoide. No presente estudo, constatou-se recidiva em 2 pacientes $(6,6 \%)$ na radiografia em AP. Carrera et al $^{15}$ descreveram 3 casos de recidiva em 21 pacientes avaliados. Alguns estudos demonstram que a perda da redução, com consequente subluxação residual da $A A C$, não afeta o resultado clínico final do tratamento. ${ }^{4,32,33}$ Cavinatto et $\mathrm{al}^{4}$ relataram um alto índice de perda da redução inicial após a fixação CC com âncoras, por via artroscópica, com resultado final satisfatório. Lädermann et al, ${ }^{34}$ em um estudo clínico, radiológico e isocinético com 37 pacientes submetidos a cerclagem CC e estabilização $\mathrm{AC}$, com suturas não absorvíveis para tratamento de LACs, encontraram perda de redução em 7 (18,9\%) pacientes, que foi relacionada aos resultados menos satisfatórios. Na presente amostra, 30\% dos pacientes tinham a impressão clínica de perda da redução, e observou-se $80 \%$ de subluxações residuais na radiografia em AP, mas a maioria dos pacientes obteve escores funcionais satisfatórios.

A padronização de critérios de avaliação radiológica dos resultados pós-operatórios é necessária. Figueiredo et al $^{35}$ descreveram a perda da redução da AC em $19 \%$ na radiografia panorâmica dos ombros. No presente estudo, empregamos a medida relativa da dCC_O/N com o intuito de medir com mais fidedignidade a perda da redução e avaliar possíveis relações entre a medida residual e os resultados funcionais insatisfatórios. Apesar de os pacientes com resultados insatisfatórios nos escores funcionais apresentarem maiores valores na razão da dCC_O/N, não houve significância estatística $(p>0,05)$. Como ambas as incidências (AP e Zanca) mostraram alguma correlação com os resultados de subluxação no valor da dCC_O/N, os autores recomendam quaisquer das incidências no seguimento radiológico pós-cirúrgico.

Ressalta-se, como característica positiva do estudo, a utilização de 3 escores (UCLA, DASH e Constant-Murley) na avaliação dos resultados funcionais, todos com resultados satisfatórios em mais de $80 \%$ dos indivíduos avaliados, além da padronização da medida da dCC_O/N como proposta para o acompanhamento pós-operatório. Por fim, a similaridade dos resultados (clínico, radiológico e funcional) entre os grupos (com ou sem transferência do ligamento $\mathrm{CA}$ ) colocou em dúvida a necessidade desta transferência adjuvante. Como principal ponto fraco, destaca-se o pequeno tamanho da amostra para se observar significância estatística entre o aumento da razão da dCC_O/N e piores resultados nos escores funcionais.

\section{Conclusão}

A técnica de tratamento cirúrgico da LAC aguda para os graus III, IV e V de Rockwood e Williams, ${ }^{3}$ com sindesmopexia CC, utilizando duas âncoras metálicas e fixação temporária clavículo-escapular, propicia uma fixação eficiente, com excelentes resultados segundo os escores de UCLA, DASH e ConstantMurley, apesar do alto índice de subluxação radiológica residual encontrada. Não foram encontradas diferenças estatisticamente significativas entre os grupos que foram ou não submetidos à transferência do ligamento CA de forma complementar no ato operatório. Resultados insatisfatórios foram associados aos indivíduos de faixa etária acima dos 50 anos.

Conflitos de Interesse

Os autores declaram não haver conflitos de interesse.

\section{Referências}

1 Court-Brown C, Heckman J, McQueen M, Tornetta P III, Ricci W, McKee M, Eds. Rockwood and Green's fractures in adults. 7th ed. Philadelphia: Wolters Kluwer Health; 2010

2 Tossy JD, Mead NC, Sigmond HM. Acromioclavicular separations: useful and practical classification for treatment. Clin Orthop Relat Res 1963;28(28):111-119

3 Rockwood C, Williams G. Disorders of the acromioclavicular joint. In: Rockwood C, Matsen F, editors. 2nd ed. Philadelphia: Saunders; 1998:483-553

4 Cavinatto L, Iwashita R, Ferreira Neto A, et al. Arthroscopic treatment of acute acromioclavicular joint dislocation using suture anchors. Acta Ortop Bras 2011;19(03):141-144

5 Johansen JA, Grutter PW, McFarland EG, Petersen SA. Acromioclavicular joint injuries: indications for treatment and treatment options. JShoulder Elbow Surg 2011;20(2, Suppl):S70-S82

6 Dal Molin D, Ribeiro F, Brasil Filho R, et al. Via de acesso cirúrgico posterossuperior para o tratamento das luxações acromioclaviculares: resultados de 84 casos operados. Rev Bras Ortop 2012;47 (05):563-567

7 Flint JH, Wade AM, Giuliani J, Rue JP. Defining the terms acute and chronic in orthopaedic sports injuries: a systematic review. Am J Sports Med 2014;42(01):235-241

8 Oku E, Andrade A, Stadiniky S, Carrera E, Tellini G. Tradução e Adaptação Cultural do Modified-University of California at Los Angeles Shoulder Rating Scale para a Língua Portuguesa. Rev Bras Reumatol 2006;46(04):246-252 
9 Orfale AG, Araújo PM, Ferraz MB, Natour J. Translation into Brazilian Portuguese, cultural adaptation and evaluation of the reliability of the Disabilities of the Arm, Shoulder and Hand Questionnaire. Braz J Med Biol Res 2005;38(02):293-302

10 Constant CR, Murley AH. A clinical method of functional assessment of the shoulder. Clin Orthop Relat Res 1987;(214): 160-164

11 Chaitanya P, Naveen P. Clinico-radiological validation of the DASH questionnaire in patients operated for fracture of shaft of humerus -. Int J Res Med Sci. 2015;3(01):287-290

12 Leite N, Faloppa F. Propedêutica ortopédica e traumatológica. Porto Alegre: Artmed; 2013

13 Doneux P, Checchia S, Miyazaki A. Padronização do estudo radiográfico da cintura escapular. Revista Bras Ortop. 1998;33 (11):883-888

14 Rush LN, Lake N, Stiefel EC, et al. Comparison of Short-term Complications Between 2 Methods of Coracoclavicular Ligament Reconstruction: A Multicenter Study. Orthop J Sports Med 2016;4 (07):2325967116658419. Doi: 10.1177/2325967116658419

15 Carrera E, Pierami R, Sugawara M, Nicolao F, Netto N, Matsumoto M. Evaluation of the surgical treatment of acromioclavicular joint dislocation with a new option for temporary fixation of the acromioclavicular joint. Tech Shoulder Elbow Surg 2013;14 (04):99-103

16 Cohen J. A power primer. Psychol Bull 1992;112(01):155-159

17 Faul F, Erdfelder E, Lang AG, Buchner A. G*Power 3: a flexible statistical power analysis program for the social, behavioral, and biomedical sciences. Behav Res Methods 2007;39(02):175-191

18 Bäthis H, Tingart M, Bouillon B, Tiling T. [Conservative or surgical therapy of acromioclavicular joint injury-what is reliable? A systematic analysis of the literature using "evidence-based medicine" criteria]. Chirurg 2000;71(09):1082-1089

19 Algarín JR, Salcedo JD, Rodríguez JO, Bello AG, Sancho FB. [Grade III acromioclavicular dislocation treated with a minimally invasive approach]. Acta Ortop Mex 2010;24(05):317-323

20 Koukakis A, Manouras A, Apostolou CD, et al. Results using the AO hook plate for dislocations of the acromioclavicular joint. Expert Rev Med Devices 2008;5(05):567-572

21 Tiren D, van Bemmel AJ, Swank DJ, van der Linden FM. Hook plate fixation of acute displaced lateral clavicle fractures: mid-term results and a brief literature overview. JOrthop Surg Res 2012; 7:2. Doi: 10.1186/1749-799X-7-2

22 Baker JE, Nicandri GT, Young DC, Owen JR, Wayne JS. A cadaveric study examining acromioclavicular joint congruity after different methods of coracoclavicular loop repair. J Shoulder Elbow Surg 2003;12(06):595-598
23 Tamaoki M, Cocco L, Pereira H, et al. Estudo transversal sobre o tratamento das lesões acrômioclaviculares agudas. Acta Ortop Bras 2009;17(05):300-304

24 Eskola A, Vainionpää S, Korkala O, Rokkanen P. Acute complete acromioclavicular dislocation. A prospective randomized trial of fixation with smooth or threaded Kirschner wires or cortical screw. Ann Chir Gynaecol 1987;76(06):323-326

25 Smith TO, Chester R, Pearse EO, Hing CB. Operative versus nonoperative management following Rockwood grade III acromioclavicular separation: a meta-analysis of the current evidence base. J Orthop Traumatol 2011;12(01):19-27

26 Leidel BA, Braunstein V, Kirchhoff C, Pilotto S, Mutschler W, Biberthaler P. Consistency of long-term outcome of acute Rockwood grade III acromioclavicular joint separations after K-wire transfixation. J Trauma 2009;66(06):1666-1671

27 Rasmont Q Delloye C, Bigare E, Van Isacker T. Is conservative treatment still defensible in grade III acromioclavicular dislocation? Are there predictive factors of poor outcome?. Acta Orthop Belg 2015;81(01):107-114

28 Guy DK, Wirth MA, Griffin JL, Rockwood CA Jr. Reconstruction of chronic and complete dislocations of the acromioclavicular joint. Clin Orthop Relat Res 1998;(347):138-149

29 Weaver JK, Dunn HK. Treatment of acromioclavicular injuries, especially complete acromioclavicular separation. J Bone Joint Surg Am 1972;54(06):1187-1194

30 Pavlik A, Csépai D, Hidas P. Surgical treatment of chronic acromioclavicular joint dislocation by modified Weaver-Dunn procedure. Knee Surg Sports Traumatol Arthrosc 2001;9(05):307-312

31 Breslow MJ, Jazrawi LM, Bernstein AD, Kummer FJ, Rokito AS. Treatment of acromioclavicular joint separation: suture or suture anchors? JShoulder Elbow Surg 2002;11(03):225-229

32 Fraser-Moodie JA, Shortt NL, Robinson CM. Injuries to the acromioclavicular joint. J Bone Joint Surg Br 2008;90(06):697-707

33 Simovitch R, Sanders B, Ozbaydar M, Lavery K, Warner JJ. Acromioclavicular joint injuries: diagnosis and management. JAm Acad Orthop Surg 2009;17(04):207-219

34 Lädermann A, Grosclaude M, Lübbeke A, et al. Acromioclavicular and coracoclavicular cerclage reconstruction for acute acromioclavicular joint dislocations. J Shoulder Elbow Surg 2011;20(03): 401-408

35 Figueiredo E, Terra B, Marczky C, et al. Avaliação do tratamento cirúrgico da luxação acromioclavicular grau III pela técnica de Weaver-Dunn modificada associada ao amarrilho coracoclavicular em atletas [acesso em 2018 jan. 25]. RBM Rev Bras Med. 2011;68(3, n.esp). Disponível em: http://www.moreirajr.com.br/revistas.asp? fase $=$ r003\&id_materia $=4595$ 